\section{Light Microscopy Criteria for Electronic Imaging}

Theodore M. Clarke, Case Corporation

Industrial laboratories have traditionally used $4^{\prime \prime} \times 5^{\prime \prime}(100 \times 125 \mathrm{~mm})$ photomicrographs. Polaroid $4^{\prime \prime} \times 5^{\prime \prime}$ prints have largely replaced glass plate and sheet film contact prints in industrial laboratories. High resolution 2000 by 3000 pixel imaging systems should be able to match the resolution of the $4^{\prime \prime} \times 5^{\prime \prime}$ photomicrographs, typically 6 cycles per $\mathrm{mm}$ at a magnification of 500 times N.A., with a significantly larger field size which better utilizes modern, wide field optics. CCD cameras with $1024 \times 1280$ pixel sensor arrays should be able to essentially match Polaroid $4^{\prime \prime} \times 5^{\prime \prime}$ photomicrograph prints in field size and resolution.

\section{INTRODUCTION}

High resolution electronic imaging is certain to be the dominant method of recording microscope images in industrial laboratories in the future. The rate at which this transition takes place will depend upon economics and the ability to match film image resolution and field size with digital imaging. This second issue is the subject of this article. The other issue, how to justify electronic imaging with a cost-benefit analysis, is outside my area of expertise. The following analysis and limited experimental results indicate that $1024 \times 1280$ pixel imaging systems should be able to match traditional 4 " $\times 5$ " photomicrographs in resolution and field size.

\section{BACKGROUND}

Ernst Abbe established that useful magnification ranges between 500 and 1000 times the numerical aperture (N.A.) of the objective lens. F.K. Moelling(1) of Carl Zeiss notes that microscopists with particularly good vision should use a criterion of 500 times the N.A. It is quite important to relate the Abbe criterion to the final image resolution. A 500 times numerical aperture photomicrograph has a theoretical maximum resolution of 6 lines or cycles/mm(2) (see Appendix). A 1.40 N.A. objective has, according to Abbe, a useful magnification range of $700 \mathrm{X}$ to $1400 \mathrm{X}$. (A higher magnification can be used for measuring or counting, but will not reveal additional detail). The diffraction limited $700 \mathrm{X}$ image, obtained with $0.55 \mu \mathrm{m}$ light, resolves 6 lines $/ \mathrm{mm}$ times 700 , or 4200 lines $/ \mathrm{mm}$ in the object. The reciprocal of 4200 lines $/ \mathrm{mm}$ is the well known $0.24 \mu \mathrm{m}$ resolution limit for the light microscope. Key detail in a photomicrograph is commonly recorded at about 3 lines per $\mathrm{mm}$ resolution, so that it is easily seen at a standard viewing distance of $250 \mathrm{~mm}$. This detail is much more faithfully recorded with a magnification criterion of 500 times N.A. than with one of 1000 times N.A. The lower magnification objectives of the compound microscope generally provide $50 \mathrm{X}, 100 \mathrm{x}$, and $200 \mathrm{X}$ images at 500 times N.A. when used with $10 \mathrm{X}$ projection or viewing eyepieces and the aperture diaphram set to fully fill the objective with illumination. The two views of Figure 1 show the loss in fine detail in the acicular ferrite zone in a metallographic specimen when a $0.10 \mathrm{~N} . \mathrm{A}$. objective is used to obtain a $100 X=1000$ N.A. photomicrograph versus $100 X=500$ N.A. with a 0.20 N.A. objective. Lower magnification images, recorded at a magnification of 500 times N.A., place greater demands on the recording medium than the $500 \mathrm{X}$ and $1000 \mathrm{X}$ images, typically recorded at 750 times the N.A. and

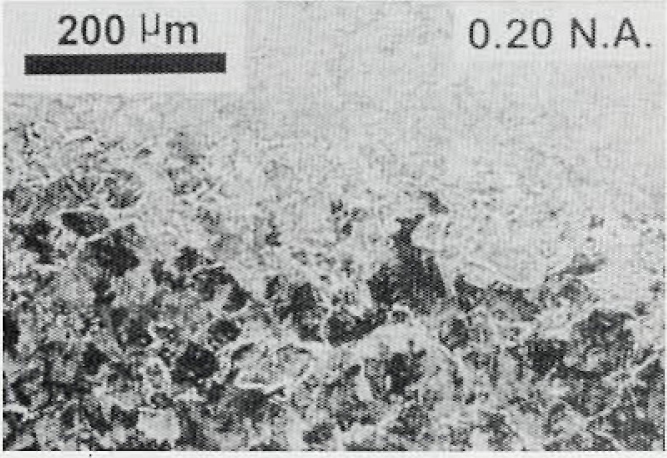

sometimes at 1000 times N.A.

The intermediate image in the compound microscopes has, until recently, covered a circle $18 \mathrm{~mm}$ in diameter. This circular field is then typically enlarged 10 times by the projection eyepiece to fill a circle $180 \mathrm{~mm}$ in diameter and recorded on $4^{\prime \prime} \times 5^{\prime \prime}$ format, which is typically Polaroid film. The maximum optical image resolution of 6 lines $/ \mathrm{mm}$, at a magnification of 500 times N.A., is easily recordable with $4^{\prime \prime} \times 5^{\prime \prime}$ Polaroid film which has a print resolution of 18 lines per $\mathrm{mm}$.

\section{PROPOSED CRITERIA OF RESOLUTION AND FIELD SIZE}

The goal of an electronic imaging system for photomicroscopy should be to record the same field size as $4^{\prime \prime} \times 5^{\prime \prime}$ Polaroid with the same resolution in object space using an Abbe criterion of 500 times N.A. This is a far easier goal to meet than trying to match $35 \mathrm{~mm}$ film recording in general photography. A 6 line per $\mathrm{mm}$ pattern in a Polaroid $4^{\prime \prime} \times 5^{\prime \prime}$ field size of $89 \times 114 \mathrm{~mm}$ could be formed by alternating the brightness of a $2 \times 6 \times 89 \mathrm{~mm}$ by $2 \times 6 \times 114 \mathrm{~mm}$ pixel array. This would require a $1070 \times 1370$ pixel array.

\section{EXPERIMENT WITH VGA IMAGING}

My initial experiment was with a $440 \times 592$ pixel Photophone system(3). A National Bureau of Standards - 1963-H Microscopy Resolution Test Chart was imaged in an $89 \mathrm{~mm}$ by $120 \mathrm{~mm}$ field size in the high resolution mode. The finest pattern that could be imaged on the monitor was 2.2 lines per $\mathrm{mm}$. The 2.5 lines per mm pattern was almost resolved as shown in the two views of Figure 2 made from a Sony Mavigraph print. The theoretical resolution limit is estimated to be $(440 \div 2) \div 89=2.5$ lines $/ \mathrm{mm}$. This is far short of 6 lines per $\mathrm{mm}$ for a $4^{\prime \prime} \times 5^{\prime \prime}$ Polaroid recorded at $1 X$ using a lens aperture of f/128 to satisfy the 500 times N.A. criterion(4). The Sony Mavigraph prints from the Photophone systems were found to match the image resolution on the monitor.

\section{EXPERIMENT WITH HIGH RESOLUTION CCD CAMERA}

Our metallurgical laboratory was very fortunate to be chosen by Carl Zeiss for a demonstration of the Roche ImageManager and Kontron ProgRes 3012 camera interfaced with an Axiovert research metallograph(5). The Kontron camera has a highest resolution mode of $3072 \times 2320$ pixels, although the monitor could display only $1024 \times 1280$ pixels. The image on the monitor showed the finest detail that could be seen through the $10 \mathrm{X}$ eyepieces. A comparison of $500 \times$ Polaroids of the same pearlitic microstructure indicated that images from the Zeiss Ultraphot used by our laboratory exhibited slightly higher resolution. The Epjplan $40 \mathrm{X}$ objective has a N.A. of 0.85 versus 0.75 on the newer, infinity corrected Zeiss objective, which has the advantages of permitting rapid change from brightfield to darkfield or DIC and a larger field of view.

Joe Barabe, of McCrone Associates, was present during the Zeiss demonstration and brought with him an Applied Image Inc. resolution test slide with a finest spacing of 228 lines $/ \mathrm{mm}$, suitable for testing low power objectives up to 0.076 N.A. The Zeiss representatives brought a $2.5 \times / 0.075$ N.A objective for use with this test pattern. The Roche system just resolved the finest pattern spacing of 228 lines $/ \mathrm{mm}$, the theoretical resolution of the objective. The system also showed one of its advantages over traditional photography: precise focus could be determined through the electronic measurement of image contrast. There was considerable difficulty in precisely focusing the $4^{\prime \prime} \times 5^{\prime \prime}$ Polaroid images because of the large depth of field of this low power objective. The best Polaroid showed the 203 line per $\mathrm{mm}$ pattern just resolved. The time available

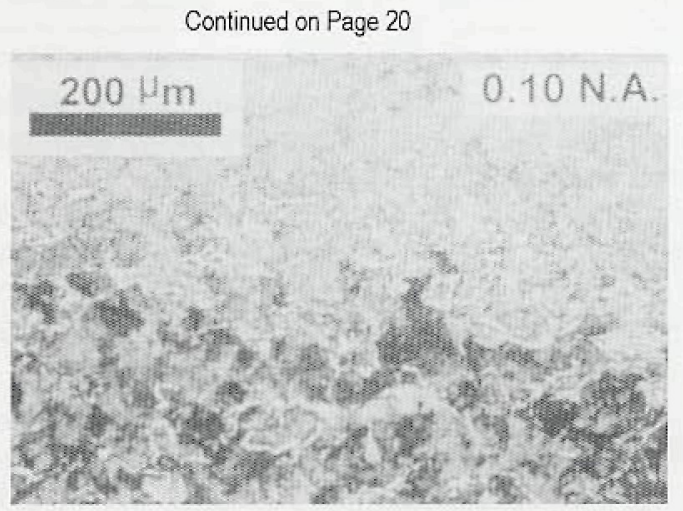

Figure 1. 100X photomicrographs (subsequently enlarged for publication) showing noticeable improvement in resolution from using a $500 \times$ N. A. criterion for magnification 


\section{Get the Most from Your Image!}

\section{The Ultimate}
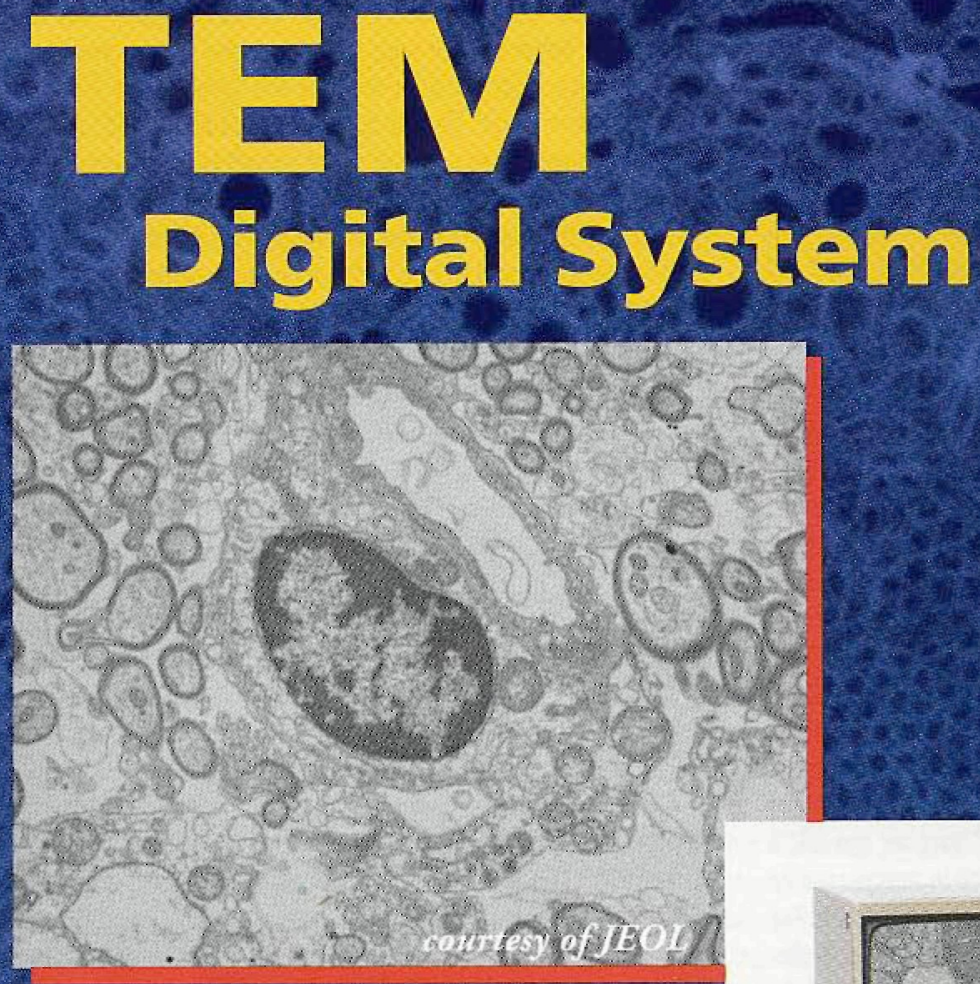

Highest Resolution

YAG Crystal Scintillator - Better

than the best phosphor

Up to $2 \mathrm{k} \times 3 \mathrm{k}$ camera resolution

Highest Sensitivity

Cooled CCD Camera

Ultra low noise electronics

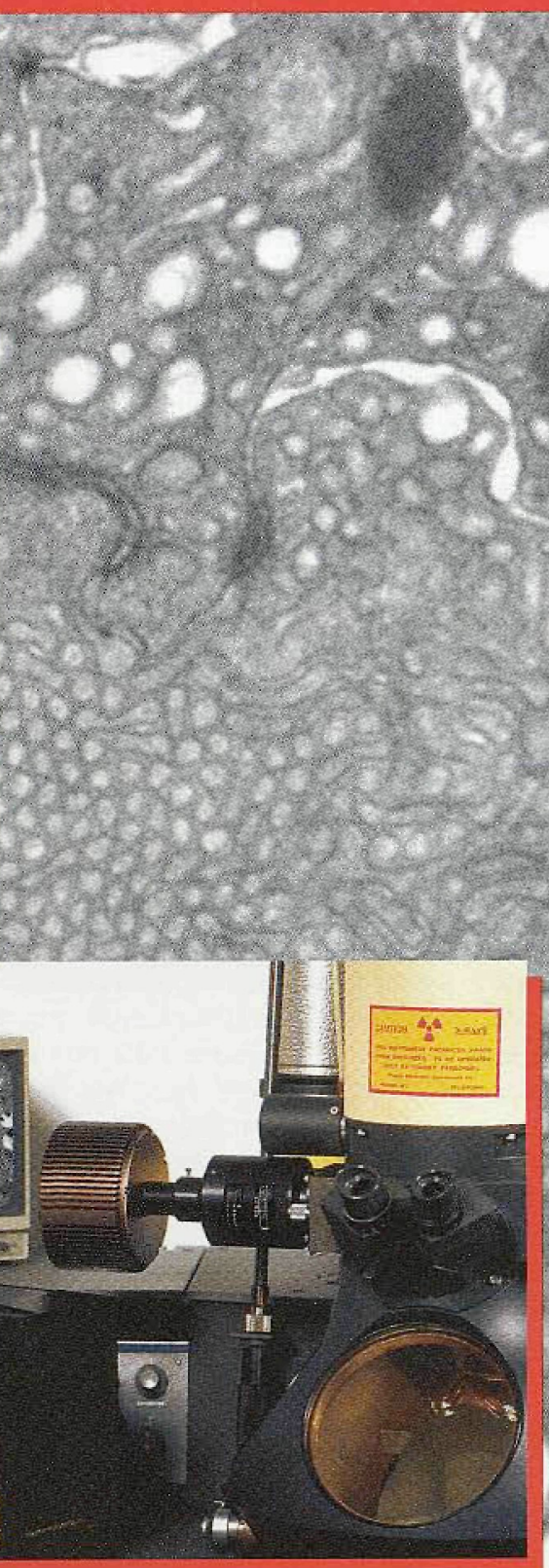

\section{Easy to use}

Unique RS-170 output

PC or MAC versions \& even most popular

werkstations

Full support for your favorite 3rd party software

SURPRISINGLY AFFORDABLE!

\section{PrincetonInstruments,inc.}

3660 Quakerbridge Road, Trenton, N.J. 08619

Tel: $609-587-9797$ Fax: 609-587-1970

Email:sales@prinst.com World Wide Web:wwwprinst.com

Worldwide representation. Call or write for the representative nearest you. 
for this testing was very limited because this was not a major reason for the Zeiss demonstration. The main factor overlooked in the test was the field size being recorded. Hard copy of the image recorded with the Roche system and stored in its hard drive was not obtained, but was requested.

Later measurements of the test patterns recorded on the Polaroid images indicated that the $4^{\prime \prime} \times 5^{\prime \prime}$ Polaroids had a magnification of about $27 X$, corresponding to a field size of about $8.9 \mathrm{~mm}$ by $11.4 \mathrm{~mm}$ in the intermediate image. The Kontron camera directly recorded the intermediate image with a recording field size of about $6.6 \mathrm{~mm} \times 8.8 \mathrm{~mm}$, or $74 \%$ of the field size recorded on Polaroid film. The 1024 by 1280 monitor displayed the 228 lines per $\mathrm{mm}$ test pattern with a field size and resolution equivalent to a $36 \times 4^{\prime \prime} \times 5^{n}$ Polaroid with a print resolution of about 6.2 lines $/ \mathrm{mm}$. The $4^{\prime \prime} \times 5^{\prime \prime}$ Polaroid was recorded at a magnification of $27 \mathrm{X}$ or 360 times the N.A., with a print resolution of 7.7 lines $/ \mathrm{mm}$. Clearly this preliminary experiment did not satisfy the goals I have proposed, but it did provide promising results and lessons for a future more carefully conducted experiment with hard copy to demonstrate the results.

\section{SUGGESTIONS FOR FUTURE EXPERIMENTS}

Since the projection eyepiece for $4^{\prime \prime} \times 5^{\prime \prime}$ Polaroid recording was $10 \mathrm{X}$, it is suggested that the next experiment be conducted with a 5X/0.10 N.A. objective, which would result in $50 \mathrm{X}$ final magnification at 500 times N.A. The Kontron camera should be used with a $0.75 \mathrm{X}$ projection eyepiece so that the field size remains the same as for the Polaroid. The resolution test chart for the experiment should have test patterns exceeding 300 lines or cycles $/ \mathrm{mm}$. (This test pattern is available on a special order from Applied Image Inc. of Rochester, NY). Assuming that the objective is diffraction limited, the $89 \mathrm{~mm}$ dimension of the 4" $\times 5$ " Polaroid recording the test pattern at 6 cycles $/ \mathrm{mm}$ is equivalent to 534 light to dark cycles (resolved lines). This would ideally require 1068 pixels to record and display, but the monitor has only 1024 pixels on the " $Y$ " axis for the 2320 possible recording pixels with the camera. Any difference in image quality between the 4" $\times 5$ " Polaroid and the $1024 \times 1280$ Pixel display on the monitor displaying the same object field size should be almost nondetectable. A $2048 \times 2048$ monitor should allow recording a far larger field size that the $4^{\prime \prime} \times 5^{\prime \prime}$ Polaroid at the same resolution. The larger field size would better utilize the wider field size available with the modern Zeiss optics. A less costly $1024 \times 1280$ pixel monitor could be used with the scrolling feature of the ImageManager to display portions of the $3072 \times$ 2320 pixel captured image, without compression, in the "unscaled" mode.

\section{CONCLUSION}

In summary, it is reasonably certain that an electronic imaging system like the Roche ImageManager with the 3200 ProgRes camera can match traditional $4^{\prime \prime} \times 5^{\prime \prime}$ photomicrographs in resolution with a larger field size than $4^{\prime \prime} \times 5^{\prime \prime}$.

\section{COMMENTS}

Microscopists will surely welcome a high resolution electronic image database system which is the main feature of the Roche system. This system can also store images from digital scanning electron microscopes. The major barriers to rapid spread in use are cost and the ability to obtain full resolution hard copies of the stored digital images at a cost similar to that of photographic prints.

My personal preference is to be able to access the images on a high resolution monitor rather than trying to find hard copies in a paper file. Transfer of the image file to a digital SEM with a $4^{\prime \prime} \times 5^{\prime \prime}$ Polaroid recording capability would be one way of obtaining high quality monochrome hard copies when necessary.

Another preference of mine would be that the $C C D$ camera have a sufficiently large sensor size to capture the fully corrected intermediate images from modern microscopes without the need for a reducing lens. This same consideration would permit optimal utilization of $35 \mathrm{~mm}$ camera macro lenses with the same camera, since most industrial laboratories also have a significant need for photomacrography along with photomicrography. A microscopic resolution test pattern and diagnostic software for the monitor and CCD camera should be periodically used to check the imaging systems and isolate the causes of any deficiencies.

1. Moellring, F.K. Microscopy from the Very Beginning; Carl Zeiss: Oberkochen, Germany, 1966

2. Clarke, T.M. "Image Field Size Limitations for Scanning Light Photomacrography"; Microscope 1993, 41, 21-30.

3. Image Data Corporation, $11550 \mathrm{HH}-10$ West, San Antonio, TX 78230-1024.

4. Clarke, T.M. "Method for Calculating Relative Apertures for Optimizing DiffractionLimited Depth of Field in Photomacrography"; Microscope 1984, 32, 219-258.

5. Roche Image Analysis Systems, 112 Orange Drive, Elon College, NC 27244.

\section{APPENDIX}

1. Object Resolution $=$

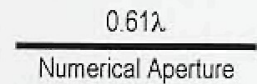

When numerical aperture (N.A.) of condenser is set equal to N.A. of objective. $\lambda=$ wavelength of light

2. Image Resolution = Magnification $\times$ Object Resolution

3. Object Resolution $=\frac{\text { Image Resolution }}{\text { Magnification }}$

4. (Combining 1. \& 3.)

5. (Setting magnification $=500$ N.A. in 4$)$

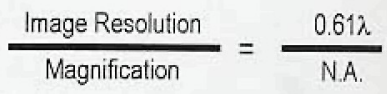

$$
\frac{\text { Image Resolution }}{500 \text { N.A. }}=\frac{0.61 \lambda}{\text { N.A. }}
$$

6. Image Resolution $=500 \times 0.61 \lambda$

7. (Setting $\lambda=5.5 \times 10^{-4} \mathrm{~mm}$ ) Image Resolution $=0.168 \mathrm{~mm}$.

$$
\frac{1}{0.168}=6 \text { lines } / \mathrm{mm}
$$

Reprinted from The Microscope, $\mathrm{Vol} 43$ No 3, 1995 from the McCrone Research Institute.

Figure 2. Photographs showing the Photophone resolution test results for a field size the same as $4^{n} \times 5^{\prime \prime}$ Polaroid ( $89 \times 114 \mathrm{~mm}$ ). 


\section{Where Else Can You Get...}
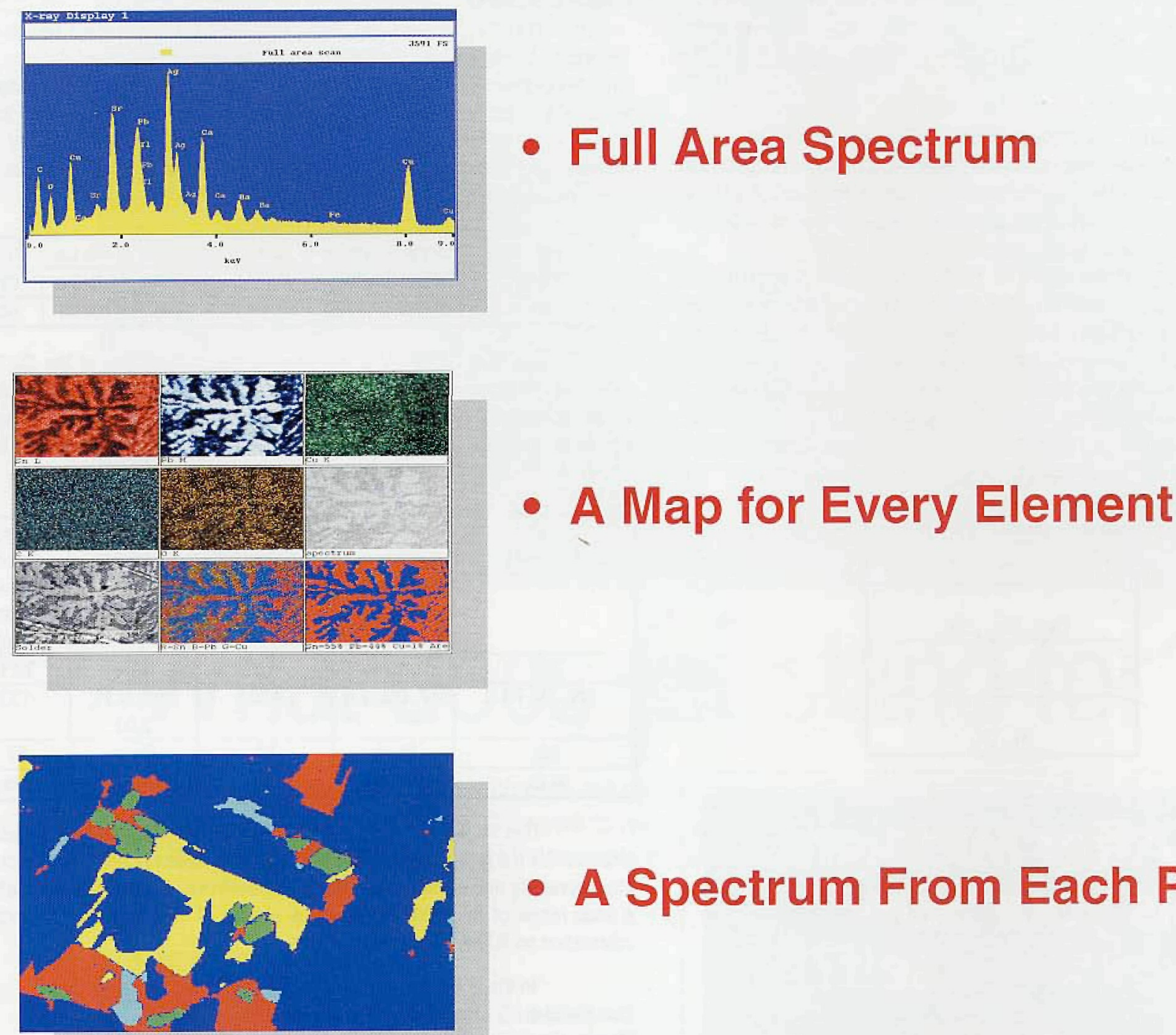

- A Spectrum From Each Phase

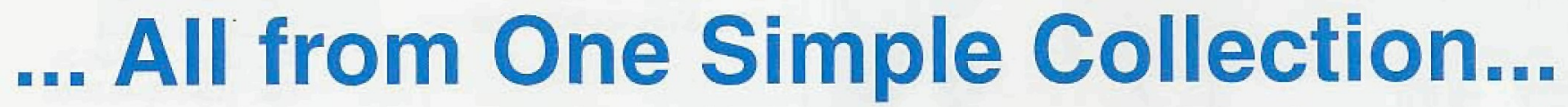

PGT introduces IMIX-PTS...Position Tagged Spectrometry. This new and revolutionary technique for X-ray microanalysis data acquisition features PGT's patented digital pulse processor. While the Electron Microscope rapidly scans the sample and acquires a high resolution electron image, $\mathrm{X}$ rays are processed and encoded with the specimen $(x, y)$ coordinate information. There is no need to perform separate collections for images, maps and spectral data or to decide beforehand what elements to map.

For more information contact PGT or visit us on the World Wide Web.

\section{See us at}

Microseopy \& Microanalysis 96

Booth \#428

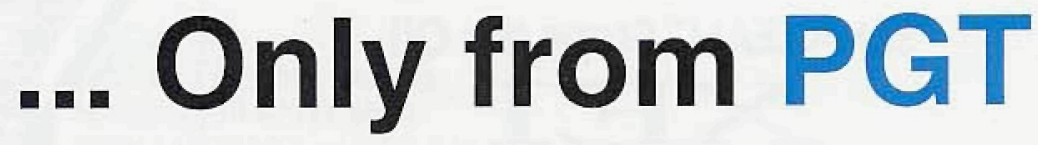

